\title{
Comparison of Posterior Hyaloid Assessment Using Preoperative Optical Coherence Tomography and Intraoperative Triamcinolone Acetonide Staining During Vitrectomy
}

\author{
Budoor Albabtain ${ }^{1,2, *}$ \\ Marco Mura ${ }^{3,4}$ \\ Patrik Schatz $\mathbb{D}^{3,5}$ \\ Sulaiman M Alsulaiman ${ }^{3}$ \\ Wael A Alsakran ${ }^{3}$ \\ Valmore A Semidey (D) $^{3, *}$ \\ 'Research Department, King Khaled Eye \\ Specialist Hospital, Riyadh, Saudi Arabia; \\ 2Department of Ophthalmology, Prince \\ Sultan Medical Military City, Riyadh, Saudi \\ Arabia; ${ }^{3}$ Vitreoretinal Division, King \\ Khaled Eye Specialist Hospital, Riyadh, \\ Saudi Arabia; ${ }^{4}$ Department of \\ Translational Medicine, University of \\ Ferrara, Ferrara, Italy; ${ }^{5}$ Department of \\ Ophthalmology, Clinical Sciences, Skane \\ County University Hospital, Lund \\ University, Lund, Sweden
}

*These authors contributed equally to this work
Purpose: To compare the specificity of diagnosing posterior vitreous detachment (PVD) using preoperative optical coherence tomography (OCT) versus intraoperative triamcinolone acetonide (TA) staining in patients undergoing vitrectomy.

Patients and Methods: This retrospective cohort study included patients undergoing pars plana vitrectomy for diverse retinal pathologies. Intraoperatively, surgeons evaluated the posterior hyaloid status with TA staining and compared it with preoperative OCT findings. Results: One hundred six patients underwent intraoperative assessments of posterior hyaloid status, with $72 \%(76 / 106)$ of the eyes showing positive staining. Sixty-two patients had also undergone preoperative OCT. Of the patients diagnosed with PVD on preoperative OCT, $50 \%(15 / 30)$ showed positive TA staining intraoperatively. The sensitivity of preoperative OCT assessment was $83.3 \%$, and its specificity was $65.9 \%$.

Conclusion: Preoperative OCT imaging is associated with lower sensitivity and specificity for diagnosing PVD when compared to intraoperative TA staining.

Keywords: optical coherence tomography, posterior hyaloid, posterior vitreous detachment, vitrectomy, vitreoschisis, triamcinolone acetonide

\section{Introduction}

The vitreous, a colorless, gel-like substance, is an extracellular matrix that plays a key role in ocular disease and health. ${ }^{1}$ In young individuals, ${ }^{2}$ the vitreous has a gel-like consistency, with strong overall attachment to the retina. ${ }^{3}$ As an individual ages, however, alterations in macromolecular interactions within the vitreous create a more liquid consistency. This liquefaction, known as syneresis, causes destabilization of the vitreous and leads to vitreous collapse and consequent posterior vitreous detachment (PVD). ${ }^{4}$

Preoperative recognition of the posterior hyaloid status helps the surgeon to better understand the surgical anatomy and allows a more precise planning of the surgical approach. ${ }^{5}$ In addition, the use of colored stains and particulate suspensions (eg, triamcinolone acetonide [TA]) intraoperatively can facilitate visualization of the multilamellar nature of the vitreous. ${ }^{6}$ Intraoperative identification and removal of the posterior hyaloid may mitigate the risk of postoperative complications, including recurrence of retinal detachment and formation of epiretinal membrane (ERM). This study aimed to compare intraoperative assessments of the posterior hyaloid presence by surgeons
Correspondence: Valmore A Semidey Vitreoretinal Division, Department of Ophthalmology, King Khaled Eye Specialist Hospital, P.O. Box 7191, Riyadh, I 1462, Saudi Arabia

Tel +966-I I-482-1234 ext.2655

Fax +966 II 482 I 234 ext.3773

Email asemidey@hotmail.com 
after triamcinolone staining with the preoperative diagnosis of PVD using spectral-domain optical coherence tomography (OCT).

\section{Patients and Methods}

This retrospective cohort study compared OCT-based preoperative diagnosis of PVD with intraoperative TA-based assessments of the presence or absence of residual vitreous or posterior hyaloid after a full vitrectomy. The study sample consisted of 106 eyes of 106 patients who had undergone intraoperative TA staining, of which preoperative OCT data of sufficient quality were available for 62 eyes. All patients were evaluated and assessed at King Khaled Eye Specialist Hospital (KKESH), Riyadh, Saudi Arabia, from January 2018 to March 2020. Patients with a diagnosis of tractional retinal detachment, vitreous hemorrhage (VH), macular hole (MH), ERM, vitreomacular traction (VMT) and those with rhegmatogenous retinal detachment (RRD) for no longer than 1 month were included. Exclusion criteria included media opacities (eg, cataract or corneal opacity), recurrent retinal detachment, and previous surgical interventions for any retinal pathology. This study adhered to the tenets of the Declaration of Helsinki. The ethics review board at KKESH granted IRB approval for this study. Informed consent was not required due to the retrospective nature of the study.

Preoperatively, patients underwent a clinical assessment that included measurements of best-corrected visual acuity using a Snellen chart, fundus examination, and applanation tonometry. Retinal structure and vitreoretinal interface were analyzed qualitatively and quantitatively using transfoveal horizontal spectral-domain OCT scans (Spectralis OCT; Heidelberg Engineering, Inc., Heidelberg, Germany) and macular volume raster scans consisting of 19 horizontal 6mm high-resolution horizontal line scans obtained within 90 days before the surgery. A trained technician performed the OCT acquisition for all eyes and two masked ophthalmologists (VAS and WAA) assessed the presence or absence of PVD from the OCT results. Disagreements between the assessors were resolved with joint review of the scans. Staging was based on a previously published OCT staging system, where stages $0,1,2$, and 3 were jointly classified as attached vitreous and stage 4 was categorized as total PVD. ${ }^{7}$

Surgery was performed using a standard three-port full vitrectomy (23-gauge). Next, intravitreal TA was administered (KENACORT OR KENALOG $40 \mathrm{mg} / \mathrm{mL}$ diluted 1:4), and surgeons evaluated the presence or absence of a posterior vitreous detachment. After TA administration, the surgeon's preferred surgical approach was used for the procedure. Any intraoperative complications were documented.

Data were collected in an Excel spreadsheet (v. 2010; Microsoft Corp., Redmond, WA, USA). Data were then cleaned, managed, and coded in Excel. Statistical analyses were performed with SPSS (v. 19.0, IBM Corp., Armonk, NY, USA). Confidence intervals were set as $95 \%$, with a corresponding $p$-value less than 0.05 was considered statistically significant. Descriptive analysis was done where categorical variables were presented as frequency (\%), while continuous variables were presented as mean (SD). Inferential analysis was then done, where intraoperative findings were correlated to the diagnoses from preoperative OCT imaging. Sensitivity and specificity analysis was conducted considering Triamcinolone staining as the gold standard. Associated sensitivity, specificity in addition to both positive and negative predictive values were calculated.

\section{Results}

The study sample included 55 males (52\%) and 51 females (48\%). At presentation, the mean age of the patients was 53 years (Table 1). The retinal pathologies requiring vitrectomy in our patients are described in Table 2. Preoperative OCT data were available for 62 patients. Among these, 32 eyes had an attached vitreous: 6 were classified as stage 0,14 as stage 1,9 as stage 2 , and 3 as stage 3 ; the 30 remaining eyes were categorized as stage 4 with a total PVD. We excluded 44/106 eyes from OCT analysis because of inadequate quality of OCT imaging (Table 3). After performing a core vitrectomy and staining with TA (Table 4), PVD was identified in 30/106 patients (28.3\%), and hyaloid attachment was noted in the remaining 76 patients $(71.7 \%)$. Of the 30 eyes graded as complete PVD on OCT, 15 (50\%) had pre-existing PVD at the time of surgery, and 15 eyes showed attached vitreous at the time of surgery (false-positive results). Of the 32 eyes graded as attached vitreous on OCT, 29 showed attached vitreous at the time of surgery and 3 showed pre-existing PVD at the time of surgery (false-negative results) (Figure 1). The sensitivity and the specificity of preoperative OCT assessment was $83.3 \%$ and $65.9 \%$, respectively. The positive predictive value was $43.6 \%$ and the negative predictive value was $92.6 \%$ assuming a disease prevalence of $24 \%$ (Table 5 ). 
Table I Patient Demographics and Previous Ocular History

\begin{tabular}{|l|l|l|l|}
\hline & & $\mathbf{n}$ & Percentage \\
\hline Age (mean \pm SD) & & 106 & $52.73 \pm 12.86$ \\
\hline Gender & Male & 55 & 51.9 \\
& Female & 51 & 48.1 \\
\hline Eye involved & Right & 59 & 55.7 \\
& Left & 47 & 44.3 \\
\hline Surgeon & Consultant & 59 & 55.7 \\
& Fellow & 47 & 44.3 \\
\hline
\end{tabular}

Table 2 Retinal Pathology for Surgical Indication of Vitrectomy

\begin{tabular}{|l|l|l|}
\hline & $\mathbf{n}$ & Percentage \\
\hline RRD & 32 & 30.2 \\
TRD & 41 & 38.7 \\
VH & 10 & 9.4 \\
VMT & 3 & 2.8 \\
ERM & 4 & 3.8 \\
FTMH & 14 & 13.2 \\
DLM & 2 & 1.9 \\
\hline
\end{tabular}

Abbreviations: RRD, rhegmatogenous retinal detachment; TRD, tractional retinal detachment; VH, vitreous haemorrhage; VMT, vitreomacular traction; ERM, epiretinal membrane; FTMH, full-thickness macular hole; DLM, Dropped Lens Material.

Table 3 Quality of OCT Results Among Study Participants

\begin{tabular}{|l|l|l|}
\hline & Frequency & Percentage \\
\hline PVD present & 30 & 28.3 \\
PVD absent & 32 & 30.2 \\
PVD could not be assessed (poor image quality) & 44 & 41.5 \\
Total & 106 & \\
\hline
\end{tabular}

Abbreviation: PVD, posterior vitreous detachment.

Table 4 Status of Posterior Hyaloid After TA Staining

\begin{tabular}{|l|l|l|}
\hline & Frequency & Percentage \\
\hline Detachment present & 30 & 28.3 \\
Detachment absent & 76 & 71.7 \\
Total & 106 & 100 \\
\hline
\end{tabular}

\section{Discussion}

The main finding in this study is that preoperative OCT may not be sufficiently sensitive to detect the status of the posterior hyaloid, which seems to imply that intraoperative TA staining is recommendable in most vitreoretinal pathologies. This confirms the findings described previously by Hwang et $\mathrm{al}^{7}$ and Stavrakas et al. ${ }^{8}$ The new approach of this study is that we, for the first time, quantified the ability of OCT to diagnose PVD, compared to intraoperative TA-assisted assessment, which allowed us to estimate the sensitivity and specificity of this OCT-based diagnosis, in a range of retinal conditions.

In retinal pathologies such as $\mathrm{MH}$, retinal detachment, proliferative vitreoretinopathy, and VMT syndrome, a partially 


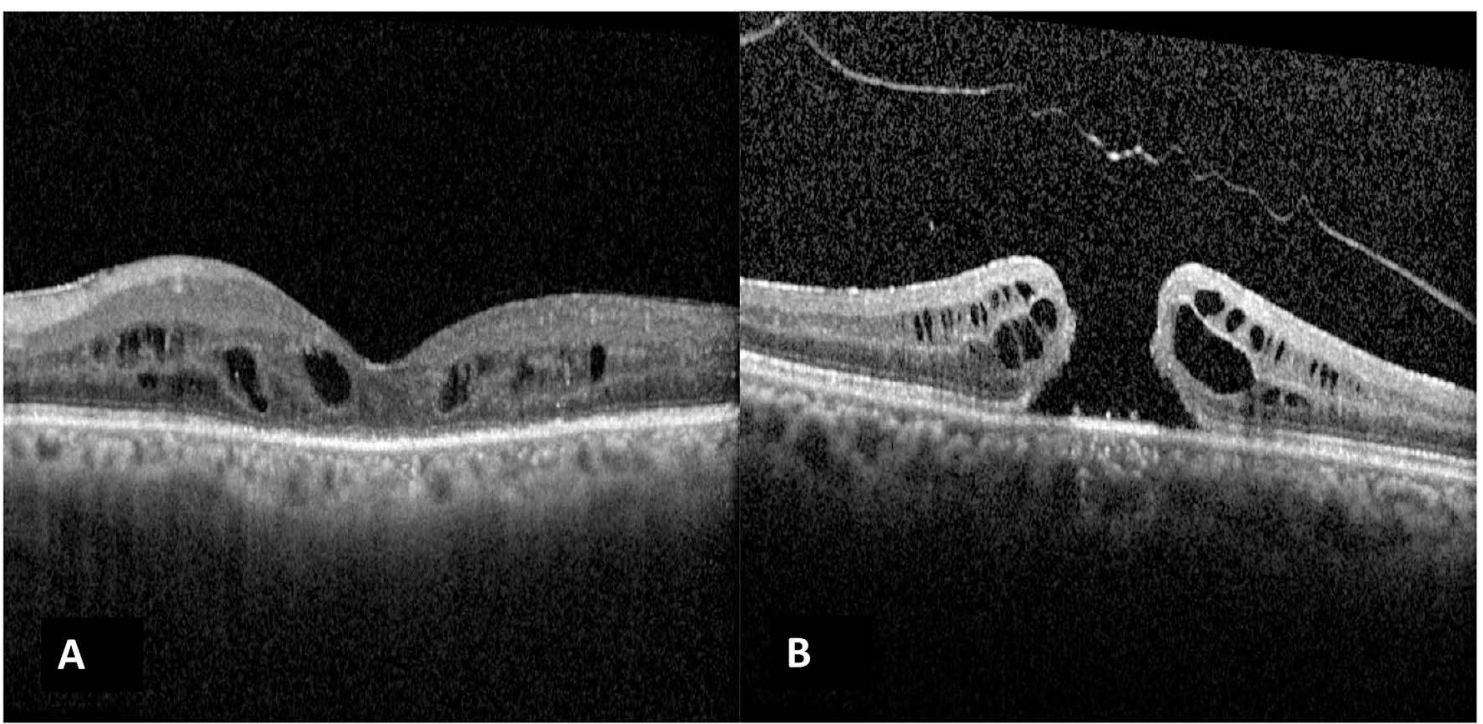

Figure I OCT image of a case labelled as Grade 4 PVD on OCT (A), but intraoperative TA staining determined a posterior hyaloid attachment (false-positive). Case (B) was labelled to have Grade 3 OCT (attached to the optic nerve head) although intraoperative staining showed that a complete PVD was present (false-negative).

separated or attached posterior hyaloid can create traction or, in patients with proliferative diabetic retinopathy, generate a framework for fibrovascular proliferation. ${ }^{9}$ In this respect, the knowledge of the posterior vitreoretinal interface anatomy is the key for a successful treatment of a large variety of surgical retinal disease. In a clinical setting, posterior hyaloid status has been found not to affect the efficacy of intravitreal injection courses for diabetic macular edema or age-related macular degeneration. ${ }^{10-12}$ However, in patients who have received therapy with intravitreal ocriplasmin for symptomatic vitreoretinal adhesion, ${ }^{13}$ ellipsoid zone disruption has been suggested to be a preexisting finding of the disease, leading to lack of visual improvement after treatment.

The outcomes of the current study indicate that a posterior vitreal attachment is common, with $71.7 \%$ (76/ 106) of patients showing this finding in association with a range of retinal baseline pathologies. The principal aim of vitrectomy is to completely remove the posterior hyaloid. Failure to achieve this step is associated with postoperative complications. A study by Yamashita et al reinforced the theory that ERMs are produced by anomalous PVDs with vitreoschisis, leaving the topmost layer of the posterior vitreous cortex attached to the macula. ${ }^{14}$ Enaida et al performed a nonrandomized study of 94 patients who underwent TA-assisted pars plana vitrectomies and 83 patients who underwent non-TA-assisted pars plana vitrectomies, reporting that $22.9 \%$ of the latter required further surgical intervention, compared to $11.7 \%$ of the TAassisted patients. ${ }^{15}$

A possible change in PVD status from the moment of OCT acquisition until surgery has been previously proposed $^{7}$ and found not to be a significant variable. Most of our patients underwent surgery shortly after OCT. Some elective cases (macular holes or ERM) did have a longer period between OCT and intervention. However, due to the chronic nature of the vitreoretinal interface pathology for these patients, we did not believe that the status of the hyaloid would change. It is also

Table 5 Overall Sensitivity and Specificity of Optical Coherence Tomography versus Triamcinolone Staining

\begin{tabular}{|l|l|l|l|l|}
\hline & & Triamcinolone Staining (Gold Standard) & & \\
\hline & & PVD Present & PVD Absent & Total \\
\hline OCT findings & PVD present & $15(83.3 \%)$ & $15(34.1 \%)$ & 30 \\
\hline & PVD absent & $3(16.7 \%)$ & $29(65.9 \%)$ & 32 \\
\hline & Total & $18(100 \%)$ & $44(100 \%)$ & 62 \\
\hline
\end{tabular}

Notes: Sensitivity $=83.3 \%$; specificity $=65.9 \%$; positive predictive value $=43.56 \%$; negative predictive value $=92.6$; assuming a disease prevalence of $24 \%$.

Abbreviations: PVD, posterior vitreous detachment; OCT, optical coherence tomography. 
noteworthy that if a preoperative OCT shows PVD, this is unlikely to change with time. False positives occurring by PVD development between the time of OCT and the surgery cannot be excluded.

Our current study demonstrated that preoperative OCT imaging can facilitate the diagnosis of PVD, although its reliability was low due to the presence of artifacts (media opacity and vitreoschisis). In the present study, 48.4\% (30/ 62) of the patients had PVD based on OCT imaging. However, TA staining confirmed that only 50\% (15) of these patients had a true PVD. On the other hand, 3 of the 32 patients who showed an attached hyaloid on OCT turned out to have complete PVD when stained by TA. This outcome indicates the low sensitivity and specificity of preoperative OCT $(83.3 \%$ and $65.9 \%$, respectively). Disease based specificity and sensitivity for PVD diagnosis on OCT was unable to calculated due to the largely uneven number of patients in each disease group (Table 2).

Several factors present challenges in assessing the status of the posterior hyaloid, such as media opacity (cataracts or $\mathrm{VH}$ ) and retinal detachment, because of which an adequate scan cannot be effectively obtained to analyze the posterior hyaloid or retinal surface. In our study, 44 patients had to be excluded from OCT analysis, due to these factors. Similar to previous findings, ${ }^{7}$ we also encountered difficulty when assessing extreme stages $(0$ or 4) of PVD. Eyes were categorized as stage 0 when the posterior hyaloid could not be clearly evaluated due to its strong adhesion to the retina, but the premacular bursa of Worst could be easily identified. Similarly, in stage 4 grading, we cannot see the posterior hyaloid due to its significant separation from the retina, but it is so far in front of the retina that the OCT cannot capture it. However, in stage 4, we would not be able to see the premacular bursa of Worst. Hwang et al compared preoperative OCT findings to operative reports describing the posterior vitreous status. ${ }^{7}$ However, triamcinolone staining was not used in $28 \%$ of their cases, leading to a wide range of possible reasons for intraoperative misdiagnosis of the posterior hyaloid, including the surgeons' level of experience, absence of TA administration, and limitations in report dictation and documentation. We decided to address all these factors by only including patients in whom TA staining had been performed and intraoperative posterior hyaloid status was documented.

The presence of ERMs could also confuse the examiner and lead to misdiagnosis. Our preoperative OCT findings mimic those obtained by Kičová et al, ${ }^{16}$ who compared biomicroscopy, B-scan ultrasound, and OCT for clinically detecting PVD preoperatively and indicated that OCT has the lowest reliability index $(12.5 \%$ of 30 eyes) for determining the correct diagnosis among all modalities. They also sought to identify minimally invasive imaging modalities that could be used reliably and efficiently in clinical settings for the detection of PVD, reporting that B-scan ultrasound was accurate but, importantly, operator-dependent. Similar observations were published by Muqit et al and Vaz-Pereira et al, ${ }^{17,18}$ who evaluated the OCT features of neovascular complexes and correlated the results of an earlier study by Chu et al. ${ }^{19}$ Gupta et al found that vitreoschisis was present in 53\% of patients with $\mathrm{MH}$, as revealed by $\mathrm{OCT}^{20}$ Recently, Stavrakas et $\mathrm{al}^{8}$ reported low sensitivity and specificity $(37.5 \%$ and $31.3 \%)$ of spectral domain OCT in patients undergoing vitrectomy for diagnosis of ERM, MH and VMT. Although several studies have attempted to describe the findings in diabetic patients with ERM on time-domain $\mathrm{OCT}^{21}$ and more recently on spectral-domain OCT, ${ }^{22}$ our description of the intraoperative findings and poor specificity of OCT are consistent with a report by Gandorfer et $\mathrm{al}^{23}$ in which 60 of 61 eyes with diabetic macular edema (98.3\%) and tangential VMT were examined with electron microscopy and showed native vitreous collagen overlying the macula. Thus, it is reasonable to conclude that the intraoperative TA-assisted technique for determining posterior hyaloid status is much more reliable than preoperative OCT imaging.

Another pathology that can lead to preoperative or intraoperative misdiagnosis is vitreoschisis, which refers to a split in the posterior vitreous cortex. Recently, combined OCT and scanning laser ophthalmoscopy (SLO) have been used to clinically identify vitreoschisis. A previous study reported that OCT/SLO detected vitreoschisis in $53 \%$ of patients with $\mathrm{MH}$ and in $43 \%$ of patients with macular pucker. ${ }^{24}$ Additionally, Schwatz et $\mathrm{al}^{25}$ retrospectively evaluated patients with late-stage proliferative diabetic retinopathy who had undergone vitrectomy over a 2year period and found positive immunohistochemical staining for type II collagen antibody, indicating the vitreal origin of the membranes. They concluded that there was a high probability that the supplemental ERMs were the posterior leaf of torn cortical gel.

There are some limitations to this study, including the diversity of retinal pathologies and the lack of preoperative OCT data for all patients. Future studies should enroll a larger sample size with lower heterogeneity in retinal 
pathologies and perform additional OCT evaluations with supplementary/comparative preoperative B-scan assessments. Additionally, a new clinical imaging method for assessing posterior hyaloid status prior to TA staining should be devised with adequate validity, reliability, and reproducibility. Furthermore, studies including the preoperative assessment with wide-field OCT viewing are warranted.

\section{Conclusion}

A key objective of vitrectomy is the complete visualization of the posterior hyaloid and vitreous cortex. The outcomes of this study indicated that preoperative OCT imaging is associated with a low sensitivity and specificity for detecting vitreoschisis and PVD. Surgeons might consider performing TA staining of the vitreous to potentially prevent complications, negative patient outcomes, and the need for reoperations.

\section{Data Sharing Statement}

The datasets used and/or analyzed during the current study are available from the corresponding author on reasonable request.

\section{Ethics Approval}

The ethics review board at KKESH granted IRB approval for this study.

\section{Consent for Publication}

Written informed consent for publication was not required due to the retrospective nature study of this study and the lack of identifiable patient data.

\section{Consent to Participate}

Informed consent was not required due to the retrospective nature of the study.

\section{Acknowledgments}

We would like to acknowledge Dr. Amhed Mousa and Dr. Abeer Ahmed for support in the statistical analysis of this manuscript.

\section{Disclosure}

The authors declare that they have no competing interests in this work.

\section{References}

1. Sebag J. The Vitreous. New York: Springer; 1989.

2. Sebag J. Macromolecular structure of the corpus vitreous. Prog Polym Sci. 1998;23:415-446. doi:10.1016/S0079-6700(97)00035-X

3. Sebag J. Age-related differences in the human vitreoretinal interface. Arch Ophthalmol. 1991;109(7):966-971. doi:10.1001/archopht.1991.0108 0070078039

4. Sebag J. The vitreous. In: Hart WM Jr, editors. Adler's Physiology of the Eye. St. Louis: Mosby; 1992:268-347.

5. Sebag J. Posterior vitreous detachment. Ophthalmology. 2018;125 (9):1384-1385. doi:10.1016/j.ophtha.2018.05.018

6. Sebag J. Vitreoschisis. Graefes Arch Clin Exp Ophthalmol. 2008;246 (3):329-332. doi:10.1007/s00417-007-0743-x

7. Hwang ES, Kraker JA, Griffin KJ, Sebag J, Weinberg DV, Kim JE. Accuracy of spectral-domain OCT of the macula for detection of complete posterior vitreous detachment. Ophthalmol Retina. 2020;4 (2):148-153. doi:10.1016/j.oret.2019.10.013

8. Stavrakas P, Christou EE, Ananikas K, et al. Sensitivity of spectral domain optical coherence tomography in the diagnosis of posterior vitreous detachment in vitreomacular interface disorders: a prospective cohort study. Eur J Ophthalmol. 2021:11206721211020644. doi:10.1177/11206721211020644

9. Peyman GA, Cheema R, Conway MD, Fang T. Triamcinolone acetonide as an aid to visualization of the vitreous and the posterior hyaloid during pars plana vitrectomy. Retina. 2000;20(5):554-555. doi:10.1097/00006982-200005000-00024

10. Özsaygili C, Küçük B, Yildirim Y. The effect of posterior vitreous detachment on aflibercept response in diabetic macular oedema. Br J Ophthalmol. 2021;105(6):800-805. doi:10.1136/bjophthalmol-2020-316155

11. Waldstein SM, Coulibaly L, Riedl S, Sadeghipour A, Gerendas BS, Schmidt-Erfurth UM. Effect of posterior vitreous detachment on treat-and-extend versus monthly ranibizumab for neovascular agerelated macular degeneration. Br J Ophthalmol. 2020;104(7):899903. doi:10.1136/bjophthalmol-2019-314661

12. Neudorfer M, Fuhrer AE, Zur D, Barak A. The role of posterior vitreous detachment on the efficacy of anti-vascular endothelial growth factor intravitreal injection for treatment of neovascular agerelated macular degeneration. Indian $J$ Ophthalmol. 2018;66 (12):1802-1807. doi:10.4103/ijo.JJO_373_18

13. Drenser KA, Pieramici DJ, Gunn JM, et al. Retrospective Study of ellipsoid zone integrity following treatment with intravitreal ocriplasmin (OZONE Study). Clin Ophthalmol. 2021;15:3109-3120. doi:10.2147/OPTH.S285464

14. Yamashita T, Uemura A, Sakamoto T. Intraoperative characteristics of the posterior vitreous cortex in patients with epiretinal membrane. Graefes Arch Clin Exp Ophthalmol. 2008;246(3):333-337. doi:10.1007/s00417-007-0745-8

15. Enaida H, Hata Y, Ueno A, et al. Possible benefits of triamcinoloneassisted pars plana vitrectomy for retinal diseases. Retina. 2003;23 (6):764-770. doi:10.1097/00006982-200312000-00003

16. Kičová N, Bertelmann T, Irle S, Sekundo W, Mennel S. Evaluation of a posterior vitreous detachment: a comparison of biomicroscopy, B-scan ultrasonography and optical coherence tomography to surgical findings with chromodissection. Acta Ophthalmol. 2012;90(4): e264-e268. doi:10.1111/j.1755-3768.2011.02330.x

17. Muqit MM, Stanga PE. Swept-source optical coherence tomography imaging of the cortical vitreous and the vitreoretinal interface in proliferative diabetic retinopathy: assessment of vitreoschisis, neovascularisation and the internal limiting membrane. Br J Ophthalmol. 2014;98(7):994-997. doi:10.1136/bjophthalmol-2013-304452

18. Vaz-Pereira S, Dansingani KK, Chen KC, Cooney MJ, Klancnik JM Jr, Engelbert M. Tomographic relationships between retinal neovascularization and the posterior vitreous in proliferative diabetic retinopathy. Retina. 2017;37(7):1287-1296. doi:10.1097/IAE.0000 000000001336 
19. Chu TG, Lopez PF, Cano MR, et al. Posterior vitreoschisis. An echographic finding in proliferative diabetic retinopathy. Ophthalmology. 1996;103(2):315-322. doi:10.1016/S0161-6420(96) 30698-2

20. Gupta P, Yee KM, Garcia P, et al. Diseases of the vitreomacular interface. $\mathrm{Br} J$ Ophthalmol. 2011;95(3):376-380. doi:10.1136/ bjo.2009.175109

21. Gaucher D, Tadayoni R, Erginay A, Haouchine B, Gaudric A, Massin P. Optical coherence tomography assessment of the vitreoretinal relationship in diabetic macular edema. Am $J$ Ophthalmol. 2005;139:807-813. doi:10.1016/j.ajo.2004.12.084

22. Ophir A, Martinez MR. Epiretinal membranes and incomplete posterior vitreous detachment in diabetic macular edema, detected by spectral-domain optical coherence tomography. Invest Ophthalmol Vis Sci. 2011;52(9):6414-6420. doi:10.1167/iovs.10-6781
23. Gandorfer A, Rohleder M, Grosselfinger S, Haritoglou C, Ulbig M, Kampik A. Epiretinal pathology of diffuse diabetic macular edema associated with vitreomacular traction. Am J Ophthalmol. 2005;139 (4):638-652. doi:10.1016/j.ajo.2004.11.035

24. Sebag J, Gupta P, Rosen RR, Garcia P, Sadun AA. Macular holes and macular pucker: the role of vitreoschisis as imaged by optical coherence tomography/scanning laser ophthalmoscopy. Trans Am Ophthalmol Soc. 2007;105:121-131.

25. Schwatz SD, Alexander R, Hiscott P, Gregor ZJ. Recognition of vitreoschisis in proliferative diabetic retinopathy. A useful landmark in vitrectomy for diabetic traction retinal detachment. Ophthalmology. 1996;103(2):323-328. doi:10.1016/S0161-6420(96) 30697-0
Clinical Ophthalmology

\section{Publish your work in this journal}

Clinical Ophthalmology is an international, peer-reviewed journal covering all subspecialties within ophthalmology. Key topics include: Optometry; Visual science; Pharmacology and drug therapy in eye diseases; Basic Sciences; Primary and Secondary eye care; Patient Safety and Quality of Care Improvements. This journal is indexed on PubMed

Submit your manuscript here: https://www.dovepress.com/clinical-ophthalmology-journal
Dovepress

Central and CAS, and is the official journal of The Society of Clinical Ophthalmology (SCO). The manuscript management system is completely online and includes a very quick and fair peer-review system, which is all easy to use. Visit http://www.dovepress.com/ testimonials.php to read real quotes from published authors. 